\title{
FACILE SYNTHESIS OF ISOXAZOLES AND PYRAZOLES FROM $\beta$-DIKETOHYDRAZONES
}

\author{
CARLOS BUSTOS ${ }^{\prime}$, EDUARDO SCHOTT ${ }^{3}$, MARCELA RÍOS ${ }^{1}$ CHRISTIAN SÁNCHEZ $^{1}$ AND \\ JUAN-GUILLERMO CÁRCAMÓ ${ }^{2}$
}

\begin{abstract}
${ }^{I}$ Instituto de Quimica, Universidad Austral de Chile, Avda. los Robles s/n, Casilla 567, Valdivia, Chile.
${ }^{2}$ Instituto de Bioquímica, Universidad Austral de Chile, Avda. los Robles s/n, Casilla 567, Valdivia, Chile.

${ }^{3}$ Departamento de Ciencias Químicas, Universidad Andrés Bello, República 275, Santiago de Chile, Chile.
\end{abstract}

(Received: January 26, 2009 - Accepted: March 17, 2009)

\begin{abstract}
New 3,5-dimethyl-4-[(E)-4-( $\mathrm{R}_{1}$-phenyl)diazenyl]isoxazoles and 3,5-dimethyl-1-( $\mathrm{R}_{2}$-phenyl)-4-[(E)-( $\mathrm{R}_{1}$-phenyl)diazenyl]-1H-pyrazoles may be obtained by reaction of 3-[2-( $\left(\mathrm{R}_{1}\right.$-phenyl)hydrazono)]pentane-2,4-dione with $\mathrm{H}_{2} \mathrm{NOH} \cdot \mathrm{HCl}$ and $\mathrm{R}_{2}-4-\mathrm{C}_{6} \mathrm{H}_{4}-\mathrm{NHNH}_{2}$, respectively. The reactions were performed in ethanol as
\end{abstract} solvent and catalyzed by glacial acetic acid.

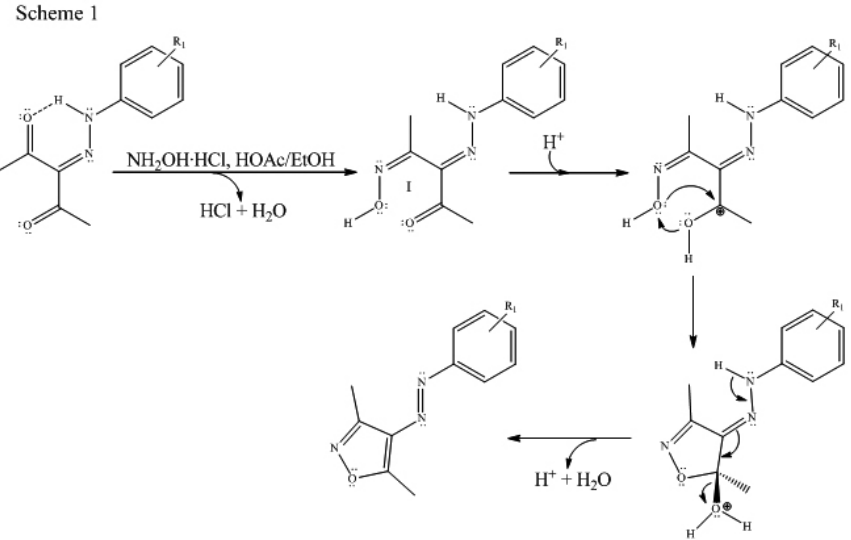

$\mathrm{R}_{1}=4-\mathrm{OH}(\mathbf{1}) ; 4-\mathrm{OCH}_{3}(\mathbf{2}) ; 4-\mathrm{COCH}_{3}(\mathbf{3}) ; 4-\mathrm{COOCH}_{2} \mathrm{CH}_{3}(\mathbf{4}) ; 4-\mathrm{H}(\mathbf{5}) ; 4-\mathrm{CH}_{3}(\mathbf{6}) ; 4-\mathrm{Cl}(\mathbf{7})$; $4-\mathrm{Br}(\mathbf{8}) ; 2-\mathrm{CO}_{2} \mathrm{H}(9) ; 4-\mathrm{CO}_{2} \mathrm{H}(10) ; 4-\mathrm{CN}(11) ; 4-\mathrm{NO}_{2}(12) ; 4-\mathrm{N}\left(\mathrm{CH}_{3}\right)_{2}(13)$

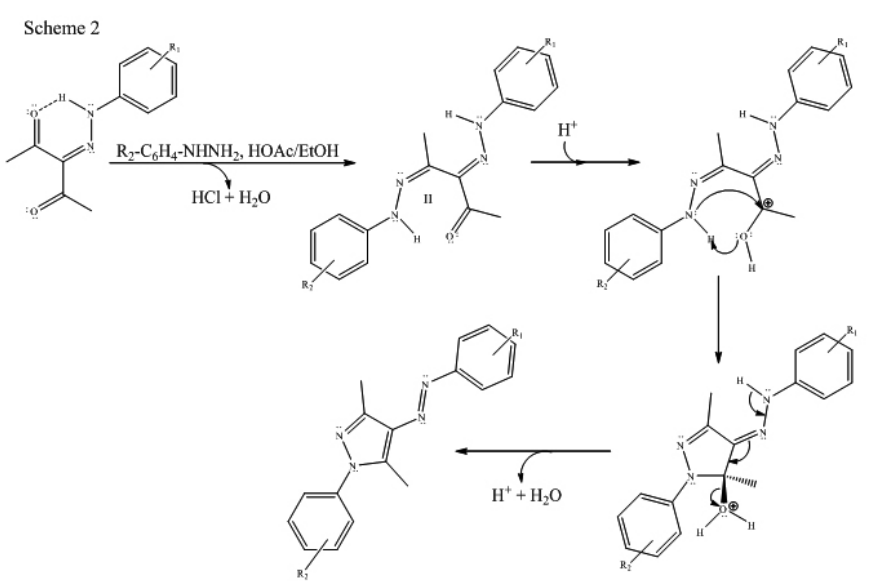

$\mathbf{R}_{1}=4-\mathrm{OH} ; 4-\mathrm{OCH}_{3} ; 4-\mathrm{COCH}_{3} ; 4-\mathrm{COOCH}_{2} \mathrm{CH}_{3} ; 4-\mathrm{H} ; 4-\mathrm{CH}_{3} ; 4-\mathrm{Cl} ; 4-\mathrm{Br} ; 2-\mathrm{CO}_{2} \mathrm{H}$;

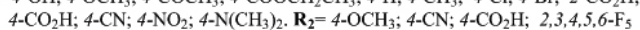

These series of compounds have been characterized using elemental analysis, gas-chromatography/mass-spectrometry and spectroscopic methods (UV-visible, IR, ${ }^{1} \mathrm{H}$ NMR, ${ }^{13} \mathrm{C}$ NMR). A typical two-dimensional HBMC spectrum of one member of the isoxazoles series, is shown in Figure 1. Besides, the crystalline and molecular structures of one isoxazole ${ }^{28}$ and two pyrazoles ${ }^{29}$ ${ }^{30}$ have been recently authenticated by X-Ray diffraction analysis. Some additional synthetic details may be found in these previous report $\mathrm{s}^{28-30}$.

Additional studies on this subject, involving other $\beta$-diketone precursors, v. gr. $\mathrm{CH}_{2}\left(\mathrm{COR}_{1}\right)\left(\mathrm{COR}_{2}\right),\left(\mathrm{R}_{1}=\mathrm{Ph}, \mathrm{R}_{2}=\mathrm{Me}, \mathrm{Ph}\right), \mathrm{CH}_{2}\left(\mathrm{COCH}_{3}\right)\left(\mathrm{CO}_{2} \mathrm{Et}\right)$ or $\mathrm{CH}_{2}\left(\mathrm{CO}_{2} \mathrm{Et}\right)$, are currently in progress whose results will be published at due time. Finally, the biological activity of isoxazoles will be evaluated as the following stage.

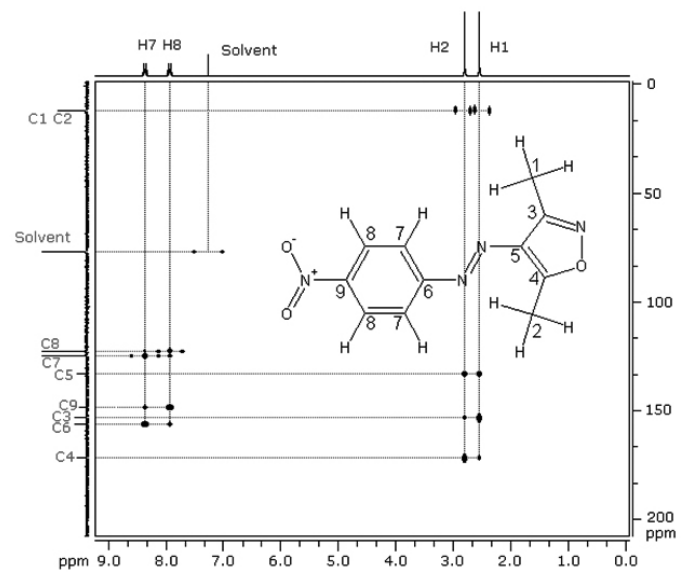

Figure 1: $\mathrm{HBMC}$ spectrum obtained in $\mathrm{CDCl}_{3}$ on a Bruker $\mathrm{AC} 200 \mathrm{P}$ Spectrometer $\left({ }^{1} \mathrm{H}\right)\left({ }^{13} \mathrm{C}\right.$ at $50.3 \mathrm{MHz}, J$ mod. sequence $\left.{ }^{1} \mathrm{H} 200.1 \mathrm{MHz}\right)$ using internal TMS. 


\section{ACKNOWLEDGMENTS}

The author greatly acknowledges the financial support received from FONDECYT (Grant 1080269) and DID-UACh, of the Universidad Austral de Chile (Grant S 2006-45).

\section{REFERENCES}

1. Dainone, G.; Raffa, D.; Maggio, B.; Plescia, F.; Cutuli, V. M.C.; Mangano, N. G.; Caruso, A. Arch. Pharm., Pharm. Med. Chem. 332, 50, (1999).

2. Bhatt, A. H.; Parekh, H.; Parikh, A. R. Heterocycl. Commun. 4, 361, (1998).

3. Talley, J. J. Prog. Med. Chem. 13, 201, (1999).

4. Talley, J. J.; Brown, D. L.; Carter, J. S.; Graneto, M. J.; Koboldt, C. M.; Masferrer, J. L.; Perkins, W. E. Rogers, R. S.; Shaffer, A. F.; Zhang, Y. Y.; Zweifel, B. S.; Seibert, K. J. Med. Chem. 43, 775, (2000).

5. Li, W.-T.; Hwang, D.-R.; Chen, C.-P.; Shen, C.-W.; Huang, C.-L.; Chen, T.-W.; Lin, C.-H.; Chang, Y.-L.; Chang, Y.-Y.; Lo, Y.-K; Tseng, H.-T.; Lin. C.-C.; Song, J.-S.; Chen, H.-C.; Chen, S.-J.; Wu, S.-H.; Chen, C.-T. J. Med. Chem. 46, 1706, (2003).

6. Rowley, M.; Broughton, H. B.; Collins, I.; Baker, R.; Emms, F.; Marwood, R.; Patel, S.; Ragan, C. I. J. Med. Chem. 39, 1943, (1996).

7. Frolund, B.; Jorgensen, A. T.; Tagmose, L.; Stensbol, T. B.; Vestergaard, H. T.; Engblom, C.; Kristiansen, U.; Sanchez, C.; Krogsgaard-Larsen, P.; Lijefors, T. J. Med. Chem. 45, 2454, (2002).

8. Haufel, J.; Breitmaier, E. Angew. Chem. 13, 604, (1974).

9. Wustrow, D. J.; Capiris, T.; Rubin, R.; Knobelsdorf, J. A.; Akunne, H.; Davis, M. D.; MacKenzie, R.; Pugsley, T. A.; Zoski, K. T.; Heffner, T. G.; Wise L. D. Bioorg. Med. Chem. Lett. 8, 2067, (1998)

10. Eid, A. I.; Kira, M. A.; Fahmy, H. H. J. Pharm. Belg. 33, 303, (1978).

11. Menozzi, G.; Mosti, L.; Fossa, P.; Mattioli, F.; Ghia, M. J. Heterocycl. Chem. 34, 963, (1997).

12. Penning, T. D.; Talley, J. J.; Bertenshaw, S. R.; Carter, J. S.; Collins, P.W.; Docter, S.; Graneto, M. J.; Lee, L. F.; Malecha, J. W.; Miyashiro, J. M.; Rogers, R. S.; Rogier, D. J.; Yu, S. S.; Anderson, G. D.; Burton, E. G.; Cogburn J. N.; Gregory, S. A.; Koboldt, C. M.; Perkins, W. E.; Seibert, K.; Veenhuizen, A. W.; Zhang, Y. Y.; Isakson, P. C. J. Med. Chem. 40, 1347, (1997).
13. Habit, N. S.; Tawil, G. G. Sci. Pharm. 49, 42, (1981).

14. Pathak, R. B.; Bahel, S.C. J. Indian Chem. Soc. 57, 1108, (1980).

15. Devi, S.; Mitro, P.; Mishra, S. B.; Mittra, A. S. J. Indian Chem Soc. 60, $679,(1983)$.

16. Daidone, G.; Maggio, B.; Plesia, S.; Raffa, D.; Musiu, C.; Milia, C.; Perra, G.; Marongiu, M. E. Eur. J. Med. Chem. 33, 375, (1998).

17. El-Emary, T. I.; Bakhite, E. A. Pharmazie. 54, 106, (1999).

18. Rathelot, P.; Vanelle, P.; Gasquet, M.; Delmas, F.; Crozet, M. P.; TimonDavid, P.; Maldonado, J. Eur. J. Med. Chem. 30, 503, (1995).

19. Tedlaouti, F.; Gasquet, M.; Delmas, F.; Timon-David, P.; Madadi, E.; Valenne, P.; Maldonado, J. J. Pharm. Belg. 45, 306, (1990).

20. Tedlaouti, F.; Gasquet, M.; Delmas, F.; Majester, B.; Timon-David, P.; Madadi, E.; Valenne, P.; Maldonado, J. Farmaco. 46, 1195, (1991).

21. Card, G. L.; Blasdel, L.; England, B. P.; Zhang, C.; Zuzuki, Y.; Gillette, S.; Fong, D.; Ibrahim, P. N.; Artis, D. R.; Bollag, G.; Milburn, M. V.; Kim, S.-H. Nature Biotech. 23 (Part 2), 201, (2005).

22. Wardakhan, W. W.; Louca, N. A. J. Chil. Chem. Soc. 52(2), 1145 , (2007).

23. Rojas R, Valderrama M, Garland M. T. J. Organomet. Chem. 689(2), 293, (2004).

24. Duprez, V.; Heumann, A. Tetrahedron Lett. 45, 5697, (2004).

25. Hermosilla, P.; Moreno, Y.; Buljan, A.; Belmar1, J.; Brovelli, F.; Peña, O.; Baggio, R. J. Chil. Chem. Soc. 51(1), 773, (2006).

26. H. C. Yao, J. Org. Chem. 29, 2959, (1964).

27. Bustos, C.; Sanchez, C.; Martínez, R.; Ugarte, R.; Schott, E.; Mac-Leod, Carey, D.; Garland, M. T. Espinoza, L. Dyes And Pigments. 74, 615, (2007).

28. Alvarez-Thon, L.; Bustos C.; Schott, E.; Sánchez, C.; Ibañez, A. Acta Crystallographica. E62, 0595, (2006).

29. Bustos, C.; Schott, E.; Mac-Leod Carey, D.; Ibáñez, A.; Alvarez-Thon, L. Acta Crystallographica. E62, o2499, (2006).

30. Bustos, C.; Sánchez, C.; Schott, E.; Alvarez-Thon, L.; Fuentealba, M. Acta Crystallographica. E63, o1138, (2007). 